\title{
Differential Spatial Representation of Taste Modalities in the Rat Gustatory Cortex
}

\author{
Riccardo Accolla, ${ }^{1,2}$ Brice Bathellier, ${ }^{1,3}$ Carl C. H. Petersen, ${ }^{2}$ and Alan Carleton ${ }^{1}$ \\ ${ }^{1}$ Flavour Perception Group, ${ }^{2}$ Laboratory of Sensory Processing, and ${ }^{3}$ Laboratory of Computational Neuroscience, Brain Mind Institute, Ecole Polytechnique \\ Fédérale de Lausanne, CH-1015 Lausanne, Switzerland
}

\begin{abstract}
Discrimination between foods is crucial for the nutrition and survival of animals. Remarkable progress has been made through molecular and genetic manipulations in the understanding of the coding of taste at the receptor level. However, much less is known about the cortical processing of taste sensation and the organizing principles of the gustatory cortex (GC). Using genetic tracing, it has recently been shown that sweet and bitter taste are processed through segregated neuronal circuitries along the gustatory pathway up to the cortical level. This is in disagreement with the evidence that GC neurons recorded in both anesthetized and behaving animals responded to multiple taste modalities (including sweet and bitter). To investigate the functional architecture of the GC in regard to taste modalities, we used in vivo intrinsic optical imaging, a technique that has been successfully applied to explore the organization of other neocortical regions. We found that four of the primary taste modalities (sweet, bitter, salty, and sour) are represented by distinctive spatial patterns but that no region was specific to a single modality. In addition, we found that two tastants of similar hedonic value (pleasant or unpleasant) activated areas with more common regions than two tastants with opposite hedonic value. In summary, we propose that these specific cortical patterns can be used to discriminate among various tastants.
\end{abstract}

Key words: taste maps; taste coding; in vivo imaging; sensory perception; intrinsic imaging; gustatory cortex

\section{Introduction}

In mammals, the process of encoding chemical information into taste perception extends from the receptor level to the primary gustatory cortex (GC) and to other multimodal areas (Rolls, 2005; Scott, 2005). GC neurons respond to chemical stimuli representing the five taste modalities, salty, sour, bitter, sweet, and umami (taste of glutamate) (Yamamoto et al., 1980; Ogawa et al., 1990; Stapleton et al., 2006) but also to textures and viscosity (Katz et al., 2001; Verhagen et al., 2004) and to the palatability (ingestion/rejection) of a tastant (Yamamoto et al., 1989). The GC also plays a role in the acquisition and retention of conditioned taste aversion (Yamamoto et al., 1994). The GC is therefore involved in complex processing of a tastant, including its identity, nonchemical properties, palatability, and memory. In this study, we investigated how different gustatory stimuli are spatially represented in the GC.

Two main theories on how tastant information is processed have been proposed (Smith and St. John, 1999). According to the "labeled-line" model, peripheral neurons best responding to a given taste modality carry the information on segregated pathways to the brain. The "across-fiber" theory says that different tastants are represented by different activity across a neuronal

\footnotetext{
Received Sept. 20, 2006; revised Dec. 22, 2006; accepted Dec. 29, 2006.

This work was supported by the Brain Mind Institute, Ecole Polytechnique Fédérale de Lausanne. We thank D. Van de Ville for his assistance to the linear model analysis, S. Simon for helpful discussion, and D. L. Buhl, S. Damak, J. Le Coutre, H. Markram, J. Poulet, and S. Simon for comments on this manuscript.

Correspondence should be addressed to Alan Carleton at the above address. E-mail: alan.carleton@epfl.ch. D0I:10.1523/JNEUROSC1.5188-06.2007

Copyright $\odot 2007$ Society for Neuroscience $\quad$ 0270-6474/07/271396-09\$15.00/0
}

population. Focusing on the GC level, consistent with the labeled-line model, a recent genetic tracing approach showed partially segregated neuronal circuitries for bitter and sweet along the entire gustatory pathway (Sugita and Shiba, 2005). Using extracellular recordings from anesthetized rats, a chemotopic organization of the primary GC was proposed (Yamamoto et al., 1985).

In contrast, other electrophysiological studies (Hanamori et al., 1998; Stapleton et al., 2006) have shown that the majority of GC neurons respond to multiple taste (including bitter and sweet) and other sensory modalities (mechanical, visceral). It has been also proposed that GC neurons carry taste information in their temporal and correlated firing patterns (Katz et al., 2001, 2002). This suggests a distributed but also dynamic model for taste coding. Although providing valuable information on firing activity of individual neurons, these electrophysiological approaches are not ideal to investigate the spatial organization of neuronal activity.

To address the issue of tastant identity and palatability representation, we conducted in vivo intrinsic optical imaging to map functional activity of a large cortical region (Grinvald et al., 1986). This method has been applied for the mapping of many sensory brain regions such as the olfactory bulb (Rubin and Katz, 1999), visual (Bonhoeffer and Grinvald, 1991; Bosking et al., 2000), somatosensory (Masino et al., 1993), and auditory (Bakin et al., 1996) cortices. Previous attempts to provide such data on the taste cortex (Yoshimura et al., 2004) must be regarded as inconclusive because the authors, although reporting segregated activation patterns after $\mathrm{NaCl}$ and sucrose stimulation, do not present any quantification supporting their claim. In addition, no 
unpalatable stimuli were used, leaving the question of cortical palatability representation unaddressed.

In this study, we found that four different taste modalities are represented by specific spatial patterns containing both distinct and overlapping regions, which can account for both segregated and across-population processing of a tastant. These cortical patterns may play a role in the discrimination among tastants.

\section{Materials and Methods}

Animals and surgery. The experiments were performed on postnatal day 19 (P19) to P29 Wistar rats (Charles River, Saint-Aubin Les Elbeuf, France). Rats were anesthetized with urethane ( $1.5 \mathrm{~g} / \mathrm{kg}$, i.p.). Heart rate, respiration rate, and lack of pain reflexes were monitored throughout the experiment. Additional urethane (20\% of initial dose) was administered if needed. The body temperature was kept between 36.5 and $38^{\circ} \mathrm{C}$ using a heating pad and a rectal probe (FHC, Bowdoinham, ME). A local anesthetic, lidocaine (Streuli, Uznach, Switzerland), was injected before any skin cut. Occasional intraperitoneal injections of $0.9 \% \mathrm{NaCl}$ solution were made to prevent dehydration.

The animal was tracheotomized using Intramedic PE160 tubing (BD Biosciences, Sparks, MD) and mounted on a custom-made stereotaxic snout frame, which was then tilted laterally by $60^{\circ}$ to have access to the right part of the face. Skin was cut and right masseter muscle, zygomatic arch, and eye were carefully removed. An oval plastic chamber was then cemented (GC Reline; GC America, Alsip, IL) to the skull to create a watertight compartment, the snout was freed to access the oral cavity, and the same cement was used to fix the animal to a new head frame by the dorsal part of the skull. A rectangular craniotomy was performed in the skull ( -2 to $+2 \mathrm{~mm}$ anterior bregma and -1 to $+2 \mathrm{~mm}$ ventrodorsally from the rhinal sulcus), keeping the dura mater intact. The chamber was then filled with transparent $1 \%$ agarose and flattened with a glass coverslip to reduce underlying brain movements, and images were collected through this agar window. All animal protocols conformed to the Swiss federal laws.

Intrinsic signal imaging. Intrinsic signals originates from different mechanisms such as changes in the physical properties of the tissue and/or changes of fluorescence or absorption of intrinsic molecules (for example, hemoglobin) (for review, see Grinvald et al., 1999). However, all of these signals can be efficiently used for functional mapping and are giving similar results (Frostig et al., 1990). This technique offers the best solution to reliably monitor activity of cortical regions with a very good spatial (but poor temporal) resolution (Grinvald et al., 1986; Frostig et al., 1990).

The cortex was illuminated with red light at $700 \mathrm{~nm}$ (bandpass, $20 \mathrm{~nm}$ ) using a stable $100 \mathrm{~W}$ halogen lamp and a light guides system. Images were acquired at $30 \mathrm{~Hz}$ for $10 \mathrm{~s}$ using the Imager $3001 \mathrm{~F}$ system (Optical Imaging, Mountainside, NJ) mounted on a custom-build macroscope [Navitar (Rochester, NY) $25 \mathrm{~mm}$ or Nikon (Tokyo, Japan) $50 \mathrm{~mm}$, bottom lenses; Nikon $135 \mathrm{~mm}, f=2.0$, upper lens]. The dimensions for a collected pixel matrix of $126 \times 126$ pixels were $2.6 \times 2.6 \mathrm{~mm}$ (with the 25 $\mathrm{mm}$ objective). The blood vessel pattern was taken using green light (546 $\mathrm{nm}$ interference filter) at the beginning of each experimental session, before setting the focal plane at $650 \mu \mathrm{m}$ below the surface to blur the surface blood vessels.

Tastant and mechanical stimulations. Taste stimuli were delivered through eight separate silicone tubes connected to a perfusion pencil made of eight independent polyimide lines. The common cone-shaped tip of the pencil was inserted for a length of $2 \mathrm{~cm}$ into the oral cavity of the animal held agape. Each tube was associated with its own pressurized fluid reservoir ( $20 \mathrm{ml}$ syringes). Starting $1 \mathrm{~s}$ after data frame acquisition, tastants were delivered at $0.3 \mathrm{ml} / \mathrm{s}$ for $3 \mathrm{~s}$, a time sufficient to flood the entire oral cavity. Tastant application was controlled by a computerdriven array of pinch valves (ValveLink 8; AutoMate Scientific, San Francisco, CA). All reagent-grade chemicals $(\mathrm{NaCl}$, sucrose, quinine- $\mathrm{HCl}$, citric acid; Sigma, St. Louis, MO) used as tastants were diluted in doubledistilled water to obtain the desired concentrations, and the solutions were prepared at least on a weekly basis.

In this study, we did not apply umami substances such as glutamate for the potential complexity of the elicited response because of its salt component and of the species differences for the glutamate taste (Yamamoto et al., 1991). The concentrations used (apart from $10 \mathrm{~mm} \mathrm{NaCl}$ ) were suprathreshold, chosen to elicit a medium-to-strong response on the peripheral nerve fibers in rats (Frank, 1991) and a consistent signal in the GC (our unpublished observations). Each tastant was applied from 6 to 28 times in the same rat. A distilled water $\left(\mathrm{dH}_{2} \mathrm{O}\right)$ rinse period of 35-45 $\mathrm{s}$ was given between two consecutive stimuli applications. Because of the preparation time before imaging $(4-5 \mathrm{~h})$ and the long rinse period, only a subset of tastants (usually two or three) could be applied to a single animal to reach a sufficient number of individual presentations for a particular solution. Using a solution of methylene blue, we observed that the papillae in the front of the tongue and in the palate were stained, whereas the circumvallate papilla was not (supplemental Fig. S2 A, B, available at www.jneurosci.org as supplemental material). We observed a stain on the lateral sides of the tongue, but we are unsure whether the foliate papillae were effectively stimulated. Therefore, the intrinsic signals obtained are mainly the result of stimulation of anterior tongue and palate receptors. However, recent studies showed that, in rats, the neural coding of taste modality depends primarily on the inputs of the facial nerve (anterior tongue and palate), whereas the glossopharyngeal projections (lateroposterior tongue) seem to be more involved in oromotor acceptance/rejection behaviors (King et al., 1999, 2003; Simon et al., 2006). In addition, all modalities are represented in the anterior part of the oral cavity (Chandrashekar et al., 2006). For these reasons, we believe that the intrinsic signals reported here are relevant for taste coding.

For mechanical stimulation, a piezo-controlled inert glass pipette $(\varnothing=$ $2 \mathrm{~mm} ; l=13 \mathrm{~mm}$; Sigmann Elektronik, Hüffenhardt, Germany) was inserted into the oral cavity and hit the tongue for $3 \mathrm{~s}$ at $10 \mathrm{~Hz}$ beginning $1 \mathrm{~s}$ after starting image acquisition. The protocol was repeated four times.

Stimulation protocols were all programmed with Matlab (The MathWorks, Natick, MA).

Data analysis. All analyses were performed using custom Matlab scripts. The images obtained for presentations of the same tastant were averaged in each individual rat. The responses were obtained dividing by the average of the first 15 blank frames of each data acquisition period (representing a respiratory cycle of $\sim 500 \mathrm{~ms}$ ). Unspecific and diffused darkening of the cortical region after stimulus application (DC shift) was treated with a two-dimensional Gaussian high-pass filter $(1 / 2 \pi \sigma=50$ pxls), whereas a temporal low-pass elliptic filter ( $f_{\text {stop }}=2 \mathrm{~Hz} ; f_{\text {pass }}=1$ $\mathrm{Hz} ; R_{\text {stop }}=50 \mathrm{~dB} ; R_{\text {pass }}=1 \mathrm{~dB}$ ) was used to reduce the effect of heart beat and respiratory artifacts.

Statistical maps for each animal were obtained by one-tailed $t$ test, the alternate hypothesis being that for each pixel the mean value in the control condition (no stimulus) was significantly greater $(p<0.05)$ than the mean in the stimulus condition. In the imaging experiments, we did not choose distilled water as the reference condition because the response water evoked differed from the ones evoked by other tastants (see Figs. 2, 4), consistent with the hypothesis that it could be considered a tastant per se (de Araujo et al., 2003; Stapleton et al., 2006). For gustatory stimulation, the $t$ test was performed on time-averaged frames over a $4 \mathrm{~s}$ period starting $1 \mathrm{~s}$ after stimulus onset. The choice of the time window was made to allow for the most consistent and strongest part of the signal (for a typical time course, see Fig. $4 C$ ). Because of the faster dynamics of the mechanical response, to allow for the most relevant part of the signal, statistics were done on time-averaged frames over a $1.5 \mathrm{~s}$ period, $500 \mathrm{~ms}$ after stimulus onset.

We also performed an alternative statistical approach that is not limited by the choice of a fixed time window but rather assesses significant responses by matching it to a temporal template of typical cortical intrinsic signal activation. We chose as a template an exponential decay function that fitted particularly well the decrease of reflectance observed. The statistical threshold was set to $p<0.001$, corrected with a Bonferroni criterion on a matrix of $126 \times 126$ pixels, which yields a false positive probability of $<1 \times 10^{-7}$ (for a detailed description, see supplemental material, available at www.jneurosci.org). The maps obtained gave similar results as the ones with the standard method [compare Figs. 5B, S1 B (available at www.jneurosci.org as supplemental material)].

In both standard and alternative method, the statistical maps were 
aligned with respect to the relative position of the middle cerebral artery (MCA) and the rhinal veins (RHVs). These maps were then merged into population maps in which the color scale indicates the consistency of the response across animals (high values meaning that the same region was activated in many rats).

Consistency index and overlap. In the overlap analysis (see Fig. $5 C-E$ ), we started from the population maps and generated new binary maps ( 1 and 0 values) for every modality by considering a threshold applied to every pixel of the map on the number of animals responding. We called this threshold the "consistency index." As an example, if one chooses a fixed consistency index of $20 \%$ (as in Fig. 5C), a pixel in the population map A will be given a value of 1 if at least $20 \%$ of the animals respond in this very same pixel. The degree of overlap between two maps $\mathrm{A}$ and $\mathrm{B}$ was computed as the ratio of the surfaces of $A \cap B$ (common activated area) over $A \cup B$ (overall activated area). Figure 5, $D$ and $E$, shows how the overlap evolves as a function of the consistency index.

In the concentration-dependent analysis, the latencies were calculated from the individual time course response to each concentration. The value on the time axis was determined by the intersection between the linear regression of the baseline and the regression line obtained by considering the curve points between 20 and $80 \%$ of the maximum response amplitude. In the overlap analysis (see Fig. $5 C-E$ ), the value representing the threshold of rats responding was rounded at the closest integer number. In Figure $5 E$ for same tastant comparison, we chose two random subpools (nine animals each) from the total pool of animals in which $\mathrm{NaCl}$ had been applied, calculated the overlap between the two, and repeated the procedure several times. A similar modus operandi was used with sucrose, citric acid, and quinine maps and the results were averaged together (black curve). The overlap values among five stimuli (four tastants and water) were averaged to obtain the different tastant curve (red in the figure).

Dye injection and histology. After exposing the GC, a pipette was filled with CellTracker Orange (Invitrogen, Eugene, OR) and inserted in tastespecific region. Ten minutes after injecting $300 \mathrm{nl}$ of the dye $(10 \mathrm{~mm})$, rats were perfused first with saline $(0.9 \% \mathrm{NaCl})$ containing heparin $(5 \mathrm{U} / \mathrm{ml})$ at $37^{\circ} \mathrm{C}$, and then with $4 \%$ paraformaldehyde (PFA) in $0.1 \mathrm{~m}$ phosphate buffer at $4^{\circ} \mathrm{C}$. Brains were harvested and postfixed for $24 \mathrm{~h}$ in $4 \%$ PFA at $4^{\circ} \mathrm{C}$, and then cut in $40 \mu \mathrm{m}$ coronal sections with a vibratome (VT1000; Leica, Wetzlar, Germany). Sections were counterstained with the fluorescent Nissl stains Neurotrace blue (Invitrogen) and imaged with a confocal microscope (TCS SP2 AOBS; Leica).

Behavior analysis. A total of six naive Wistar rats (four females and two males; P22 at the beginning of the test) were used as subjects. Because no gender difference was observed, all data were pooled. The rats were placed on a $12 \mathrm{~h}$ light/dark cycle, and all behavioral training was conducted during daytime. During the training period, animals had ad libitum access to food but were on a water-restricted access schedule designed to sufficiently motivate them to sample from the drinking spout. Continuous water restriction was never longer than $12 \mathrm{~h}$, and at the end of an experimental day the rats were given $10 \mathrm{~min}$ ad libitum access to water to keep them at $>85 \%$ of their baseline body weight. All animal care and procedures were in accordance with the Swiss federal laws.

Rats were placed individually in a custom-made gustometer allowing the delivery of different taste solution at the same licking spout. The final spout contained eight separate delivery lines, made with inert quartz tubing of $250 \mu \mathrm{m}$ inner diameter The application was made by opening computer driven pinch valves controlled by custom software programmed in Igor (WaveMetrics, Lake Oswego, OR). The rat initiated each trial by breaking a light beam at the sampling port opening (see Fig. $6 A$ ). One second after trial start, one pinch valve opened for $500 \mathrm{~ms}$ leading to the delivery of a drop $(\sim 4-5 \mu \mathrm{l})$ of one taste solution $(500 \mathrm{~mm}$ $\mathrm{NaCl}, 500 \mathrm{~mm}$ sucrose, $10 \mathrm{~mm}$ citric acid, $20 \mathrm{~mm}$ quinine, and $\mathrm{dH}_{2} \mathrm{O}$ ). Each trial induced the presentation of a new taste and the next trial was initialized at least $2.5 \mathrm{~s}$ after the end of the delivery of the last stimulus. In this behavioral paradigm, we monitored the licking behavior of the rats throughout the trial duration (licking the tube closed an electrical circuit). We chose distilled water, the most neutral liquid stimulus among the five, to evaluate preference or avoidance. Therefore, the licking patterns for the different tastants were all normalized to the water licking pattern. Using this simple paradigm, the hedonic value of a particular taste solution could be easily monitored. A significant increase of the licking rate with respect to the baseline (represented by the licking pattern during the first second of the trial, before stimulus application) was associated with attraction, whereas a significant decrease of the licking rate reflected repulsion (paired $t$ test analysis).

\section{Results}

\section{Functional localization of the GC}

The GC is located in the insular cortex (Kosar et al., 1986) (Fig. 1), anterior and dorsal to areas receiving visceral and nociceptive inputs (Cechetto and Saper, 1987; Barnett et al., 1995). The MCA and the RHVs provide anatomical landmarks helping to locate precisely the GC (Yamamoto et al., 1985; Kosar et al., 1986). The MCA passes vertically through the GC, whereas the RHV runs in the rhinal sulcus, perpendicular to the MCA and ventral to the GC (Fig. 1A). Despite the very lateral location of the GC (Fig. $1 B$ ), we exposed for the first time the region of interest in anesthetized rats and imaged tastant-evoked signals.

Applying different tastant solutions on the tongue consistently activated a region in the GC, whereas there was no obvious signal when no stimulus was applied (Fig. 2A). Furthermore, in this particular example, application of distilled water failed to elicit a response. We used these images to generate maps for water or taste stimuli in which, with respect to the no-stimulus condition, only statistically significant pixels were plotted (see Materials and Methods). Thus, for each rat, we obtained a statistical map. To look for regions consistently activated across different animals, using the MCA and the RHVs as reference landmarks we aligned and grouped all statistical maps ( $n=27$ rats). We confirmed at a population level that tastants consistently activated a region of $\sim 3 \mathrm{~mm}^{2}$ in the GC (Fig. $2 B$, right). Interestingly, in several rats water application elicited a response in a caudal region (Fig. $2 B$, left).

To verify that the signals were indeed coming from the GC, we injected a red dye in the regions in which we repeatedly observed taste signals ( $n=3$ rats) (Fig. $2 C$ ). After perfusing the animals and processing the brain, we confirmed the presence of the dye in the gustatory cortex $(n=3)$ (compare Figs. $2 C, 1 C$ ). These data show that tastant applications trigger neural activities that can be consistently imaged in the GC in vivo.

\section{Specificity of taste versus somatosensory response}

To test whether activation of the GC was the result of taste stimulation or was partly a consequence of the mechanical stimulation caused by liquid delivery on the tongue, we exposed the same 

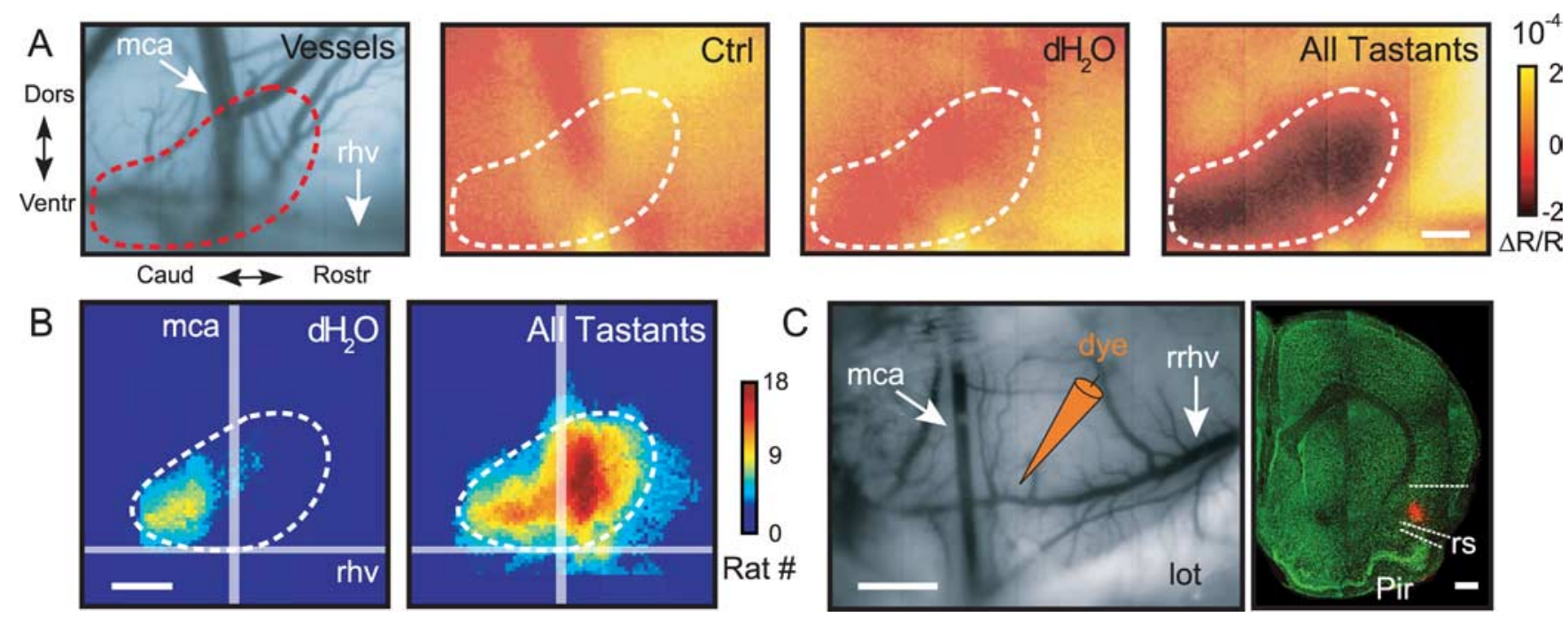

Figure 2. In vivo imaging of taste evoked response in the gustatory cortex. $A$, Left, Vascular pattern taken with a $546 \mathrm{~nm}$ illumination filter. The following images represent intrinsic responses averaged over 36 presentations of control (no stimulus), distilled water, and tastants of different modalities. Frames are averaged over a period of $2 \mathrm{~s}, 1 \mathrm{~s}$ after stimulus onset. Tastant-activated area is outlined and reported in all images. Scale bar, $500 \mu \mathrm{m}$. B, Interanimal variability analysis of the cortical regions activated by tastants. Population maps $(n=27)$ of statistically responsive pixels to distilled water and to the average of all tastants responses are shown. The color scale gives a measure of the consistency of the response across animals (high values meaning that the same region was activated in many rats). Scale bar, $1 \mathrm{~mm}$. C, Anatomical localization of the responsive area. Injection of a red dye in the taste-activated cortex before perfusion and sectioning of rat brain. The red spot in the coronal section counterstained with fluorescent Nissl stain (green) is localized within the insular cortex. rrhv, Rostral rhinal vein. See Figure 1 for other abbreviations. Scale bar, $1 \mathrm{~mm}$.
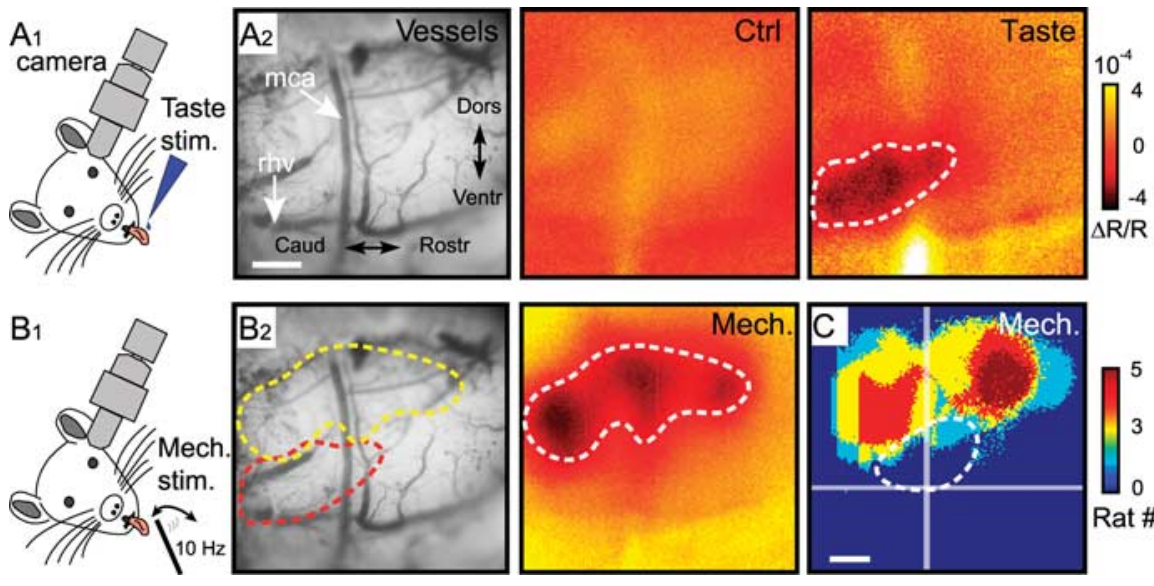

Figure 3. Signals imaged in the gustatory cortex are taste specific. $A_{1}$, Schematic drawing of the taste stimulation system. $A_{2^{\prime}}$ Intrinsic response averaged over all control and tastant presentations (144 and 24, respectively). Frames are averaged over a period of $2 \mathrm{~s}$ from response onset. $\boldsymbol{B}_{1}$, Schematic drawing of the mechanical stimulation system. $\boldsymbol{B}_{2}$, Response after mechanical stimulation of the tongue (120 averaged presentations; frames averaged $1.5 \mathrm{~s}$ from response onset) in the same animal as in $\boldsymbol{A}$. Outlined (in red and yellow) over the vessel pattern are the two nonoverlapping regions activated by taste and mechanical stimulation. $C$, Interanimal variability analysis of cortical regions activated by mechanical stimulation of the tongue $(n=5)$. The white outline (reported from Fig. $2 B$ and rescaled) shows that the separation between gustatory and somatosensory responsive areas is consistent across animals. Scale bar, $1 \mathrm{~mm}$. stim., Stimulation; Dors, dorsal; Ventr, ventral; Caud, caudal; Rostr, rostral; Mech., mechanical. See Figure 1 for other abbreviations.

animals to both taste and mechanical stimuli $(n=5$ rats). We first imaged the response to different tastants (Fig. $3 A_{1}$ ), localizing the evoked response in the GC (Fig. $3 A_{2}$ ). In the same animal, we mechanically stimulated the tongue using a glass capillary actuated by a piezoelectric (Fig. $3 B_{1}$ ). This protocol induced a response originating in a region dorsal to the GC, reflecting the somatosensory representation of the tongue and the orofacial region (Remple et al., 2003) (Fig. $3 B_{2}$ ). After mechanical stimulation, no relevant signal was observed in the GC. This is shown in the population map obtained from the five rats (Fig. 3C). Conversely, no signal attributable to taste stimulation was observed in the above-noted somatosensory area. Finally, we confirmed the taste specificity of our signals in experiments in which the chorda tympani nerve (innervating taste buds of the anterior part of the tongue and the anterior foliate papillae) was bilaterally sectioned (supplemental Fig. S2C, available at www.jneurosci.org as supplemental material). We observed that the signal induced by tastant stimulation was abolished after sensory nerve cut. This is seen in the histograms of Fig. S2D, in which the amplitude of the signals dramatically decreases after the cut, when compared with the situation before the nerve cut (before, $-7.3 \times 10^{-5} \pm$ $1.33 \times 10^{-5}$; after, $-9.8 \times 10^{-6} \pm 1.2 \times$ $10^{-5} ; n=3$ rats; paired $t$ test, $\left.p<0.05\right)$.

Together, these results do not rule out a possible somatosensory contribution to the response of our taste stimulation but the slow dynamics of intrinsic signals could not allow us to separate the somatosensory and the chemosensory part of the gustatory response as assessed by electrophysiological recordings in awake animals (Katz et al., 2001). We could nevertheless conclude that responses produced by our liquid-delivery system were taste related and were not the result of a pure mechanical stimulation. The segregation of the responses attributable to two different sensory modalities is also an additional confirmation of how optical intrinsic signals reliably monitor the activation of functionally distinct neuronal populations.

\section{Concentration dependence of taste response}

We next addressed the issue of the consistency of cortical response for a specific taste. In particular, we explored how the concentration of the stimulus affects its cortical representation. This was accomplished by exposing a group of animals $(n=7)$ to 

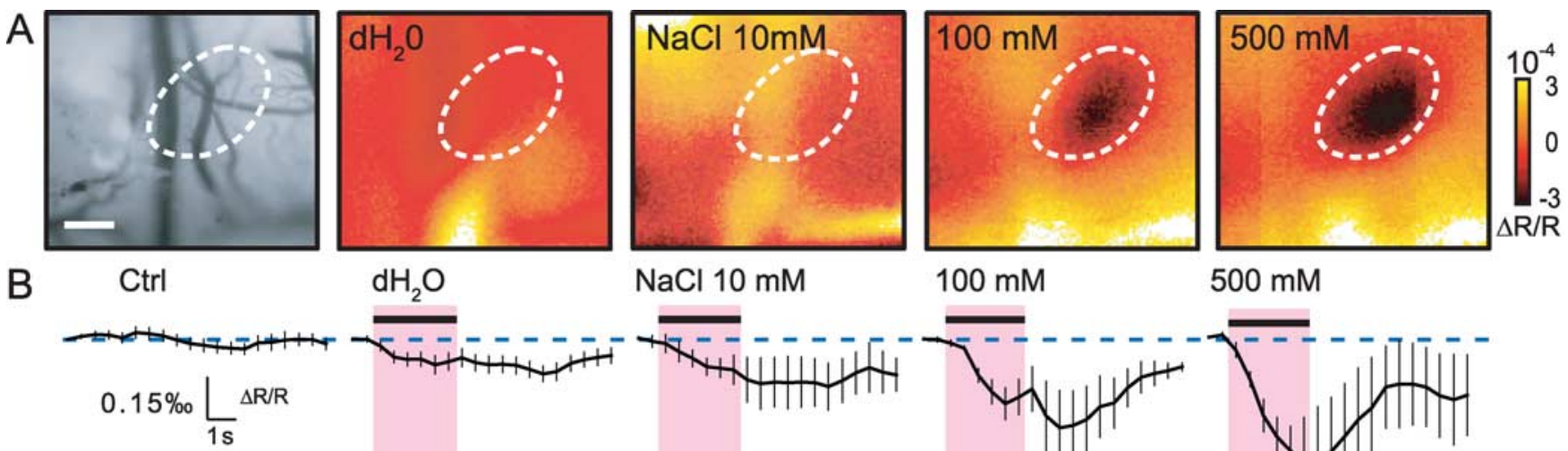

$100 \mathrm{mM}$

$500 \mathrm{mM}$
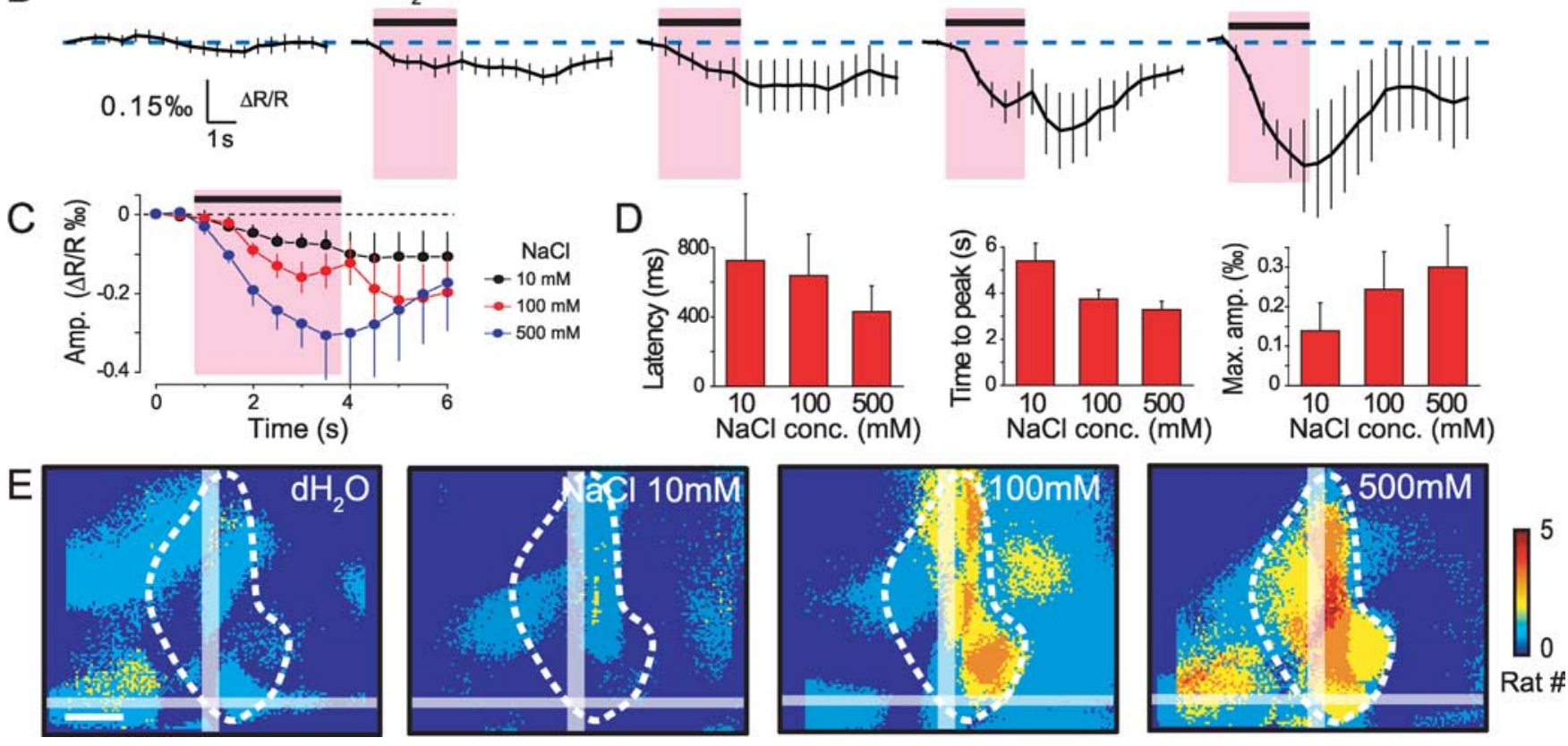

Figure 4. Analysis of concentration-dependent response to $\mathrm{NaCl}$. $A$, Example of intrinsic response to three concentrations of $\mathrm{NaCl}$ (average of 6 presentations each). Frames are averaged over a period of 2 sfrom response onset. The white outline is traced with respect to the $500 \mathrm{~mm}$ response. Scale bar, $500 \mu \mathrm{m}$. $\boldsymbol{B}$, Time course of responses to control, $\mathrm{dH} \mathrm{H}_{2} \mathrm{O}$, and $\mathrm{NaCl}$ averaged across animals $(n=7)$. The black bar above the curves indicates the duration of stimulus application. $\boldsymbol{C}$, Superposition of the dose curves represented in $\boldsymbol{B}$ at an expanded timescale. $\boldsymbol{D}$, Histograms across animals representing from left to right: decreasing latencies (response time onset), decreasing average time to reach response peak, and increasing maximum amplitude of the response with increasing concentrations. Values $(\boldsymbol{B}-\boldsymbol{D})$ are means \pm SEM. $\boldsymbol{E}$, Population maps $(n=7)$ of statistically responsive pixels. Note the stereotyped regions activated between animals at several concentrations. Scale bar, $500 \mu \mathrm{m}$.

three increasing concentrations of $\mathrm{NaCl}$, which for an awake behaving rat were close to threshold $(10 \mathrm{~mm})$, moderately salty $(100$ $\mathrm{mM}$ ), and salty (500 mM) (Contreras and Catalanotto, 1980). As a result, we found that activated areas were centered on the same cortical regions, although the amplitude and the extent of the responses increased with concentration (Fig. 4A). We quantified the concentration-dependent responses across animals by comparing the time course of the response in the activated regions (Fig. $4 \mathrm{~B}$ ). The absolute amplitude of responses increased (from 10 to $500 \mathrm{~mm}: 1.38 \times 10^{-4} \pm 0.72 \times 10^{-4}, 2.44 \times 10^{-4} \pm 0.95 \times$ $10^{-4}$, and $\left.3.00 \times 10^{-4} \pm 1.04 \times 10^{-4} \Delta R / R\right)$, whereas the time to peak decreased at higher concentrations $(5.40 \pm 0.76,3.75 \pm$ 0.43 , and $3.29 \pm 0.36 \mathrm{~ms}$ ), indicating that the GC responds in proportion to gustatory stimulus intensity (Fig. $4 C, D$ ). The latency of the response also exhibited a decrease with increasing concentrations $(10 \mathrm{~mm}, 725 \pm 378 \mathrm{~ms} ; 100 \mathrm{~mm}, 638 \pm 234 \mathrm{~ms}$; $500 \mathrm{~mm}, 431 \pm 148 \mathrm{~ms}$ ). Population maps for the three salt concentrations ( $n=7$ rats) (Fig. $4 E$ ) indicate that the extent of activated regions is more consistent across animals when the concentration is far above the detection threshold (Fig. $4 E$, white outline). A small response in the outlined salt-specific region (vertically, rostral to the MCA) is also present in two animals after the application of the lowest salt concentration (10 mM).

\section{Maps of taste modalities}

We next studied the spatial representation of the four basic taste modalities, salty, sweet, sour, and bitter in the GC. Intrinsic signal maps showed that, in the same rat, $500 \mathrm{~mm}$ sucrose (sweet) and 20 $\mathrm{mm}$ quinine (bitter) stimuli induced responses in regions that were quite distinct (Fig. 5A, top). In another example, two other taste modalities (salty and sour) are compared and the responses show again two differential patterns of activation (Fig. $5 \mathrm{~A}$, bottom). These observations were further confirmed for a large number of animals. We tested four stimuli: $\mathrm{NaCl}$ (500 mm; $n=$ 18 animals), sucrose ( $500 \mathrm{~mm} ; n=15)$, citric acid ( $10 \mathrm{~mm} ; n=8)$, and quinine ( $20 \mathrm{~mm} ; n=8$ ). After performing imaging experiments, population maps were generated. The resulting maps for each individual taste modality were quite stereotyped, because the animals tended to display responses in the same region of the GC (Fig. 5B). Importantly, the different taste stimuli elicited distinct spatial domains of activation. The population maps are the result of statistical analyses performed on time-averaged frames over a window representing the most consistent and strongest part of the response (see Materials and Methods and Fig. $4 C$ for typical activation time course). As a check, we used, as an alternative analysis, a linear parametric test that compares the temporal evolution of the intensity of a pixel to the typical intrinsic 

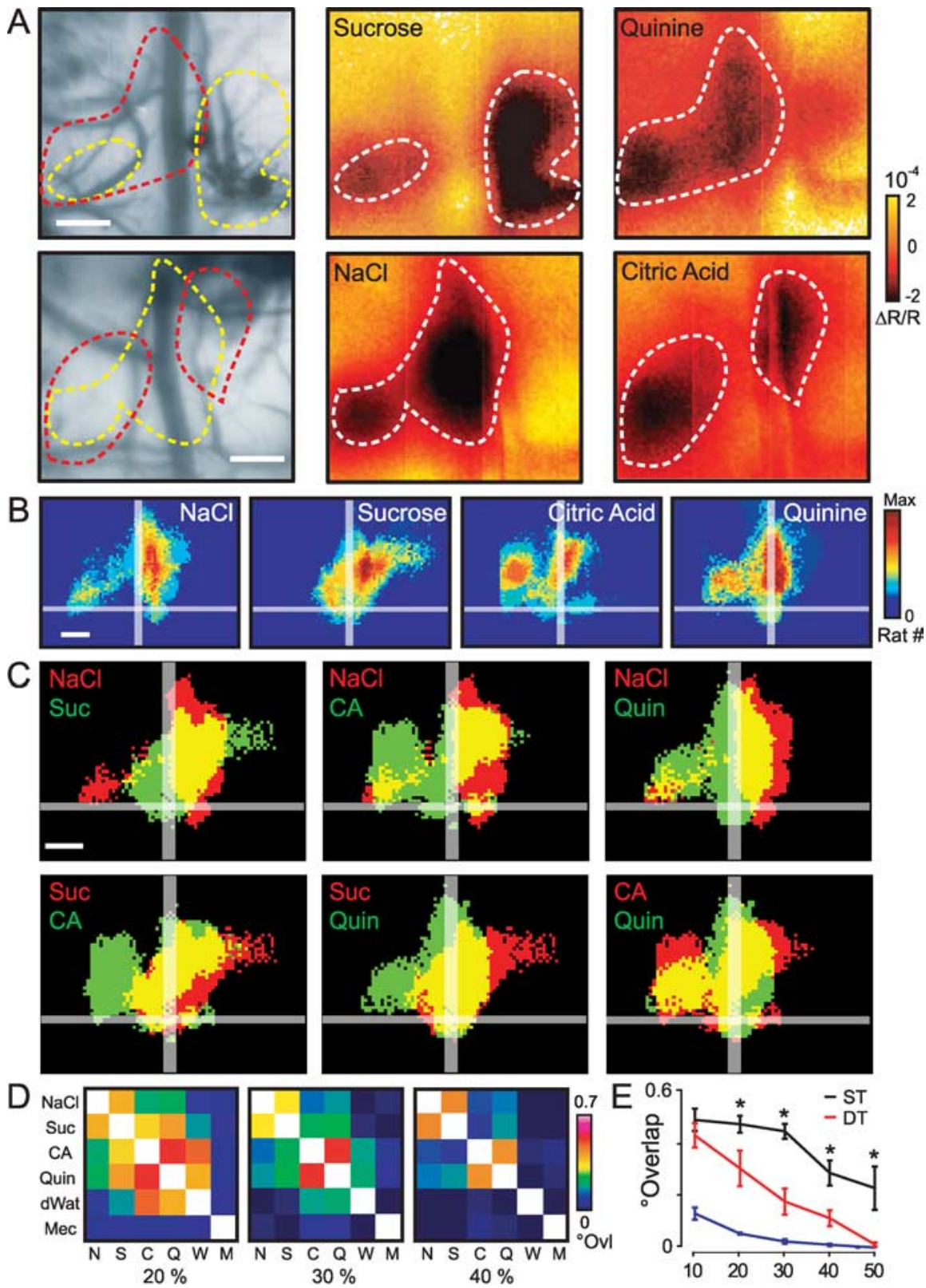

Consistency index
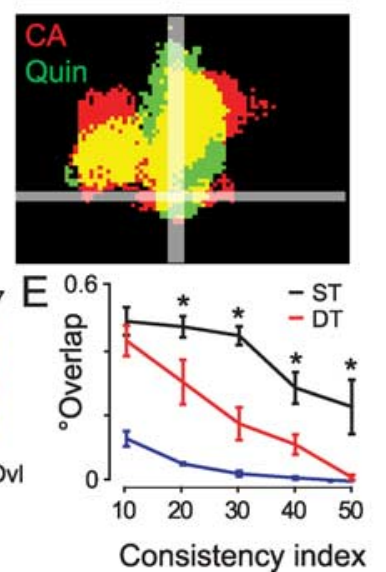

Figure 5. Differential activation patterns of tastant modalities. $\boldsymbol{A}$, Two different examples of activation maps after sucrose and quinine stimulation (top) and $\mathrm{NaCl}$ and citric acid stimulation (bottom). Twenty-eight presentations of each stimulus are averaged. Note that the some regions activated are clearly different. $\boldsymbol{B}$, Population maps for the four basic taste modalities, $\mathrm{NaCl}(n=$ $18)$, sucrose $(n=15)$, citric acid $(n=8)$, and quinine $(n=8)$. Imaging was done on a total of 27 animals, testing at least two tastants chosen randomly among the four. Color scale "Max" represents $60 \%$ of total number of animals. C, Comparison of activated areas between tastants. The yellow areas represent overlapping regions. Single stimulus surfaces are derived from $\boldsymbol{B}$ considering pixels responding with a consistency index of $20 \%$. Scale bars, $500 \mu \mathrm{m}$. D, Matrices showing the relative degree of overlap ( $\left.{ }^{\circ} \mathrm{Ovl}\right)$ between stimuli. The three matrices are computed from different consistency indices $(20,30$, and 40\%). Color-coded values are the result of the ratio between the overlap area (yellow in $C$ ) and the total activated area. The value for the white squares is 1. N, NaCl; Suc, S, sucrose; CA, C, citric acid; Quin, Q, quinine; dWat, W, distilled water; Mec, M, mechanical. E, Overlap curves as a function of the consistency index. Mean overlap values among same tastants pairs (ST) (black), different tastants pairs (DT) (red), and between mechanical stimulation and tastants (blue). Difference between black and red line is statistically significant using ANOVA ( ${ }^{*}$ Newman-Keuls post hoc test, at least $p<0.0004$ ).

signal time course [see Materials and Methods and supplemental material (available at www.jneurosci.org)]. The resulting population maps are similar to the ones obtained with the standard procedure (supplemental Fig. S1, available at www.jneurosci.org as supplemental material). Independent of the two methods

adopted, the maps evoked by different tastants also show overlapping regions that are consistent with the existence of broadly tuned GC neurons (Hanamori et al., 1998; Stapleton et al., 2006). These results can be visualized if the population maps for two tastants are overlaid with the area responding to one tastant in green, the other in red, and the overlapping region in yellow (Fig. 5C). These overlay plots were obtained from the population maps by using a threshold on every pixel (indicating the number of animals responding) that we called "consistency index" (see Materials and Methods) because it reflects the reproducibility of the activated area across animals.

A quantification of the overlap was performed by comparing one-by-one the population maps resulting from all different stimuli applications (four tastants, Fig. $5 B$; distilled water, Fig. $2 B$; mechanical, Fig. $3 C$ ). The results are presented in matrices in which the color code reflects the degree of overlap between two maps ( 0 being no overlap and 1 being complete overlap) (Fig. 5D). The three matrices correspond to the overlaps computed for different consistency indices. First, low overlap values confirmed that taste and mechanical stimulation activate segregated regions (as shown in Fig. 3C). Furthermore, different taste modalities activated different GC regions because the degree of overlap was usually $<50 \%$ and dramatically decreased when increasing the threshold (i.e., resulting in a more stereotyped map) (Fig. 5D). We then plotted the averaged overlapping degree between stimuli as a function of the consistency in$\operatorname{dex}$ (Fig. 5E). The overlap between different tastants was significantly higher than the one between tastants and water $\left(\right.$ ANOVA; $F_{(1,8)}=9.4 ; p<0.02$ ) (data not shown) or taste and mechanical maps $\left(F_{(1,9)}=40.9 ; p<2 \times 10^{-4}\right)$. This analysis quantified the region specificity for gustatory over pure mechanical response. As a control for interanimal variability, we generated two population maps for the same taste modality by randomly selecting subpools of animals and computed their degree of overlap (see Materials and Methods). We found that a match between same tastant maps was significantly greater than between different taste modality maps $\left(F_{(1,30)}=9.3 ; p<0.005\right)$ (Fig. $5 E$, compare black and red curves). This outcome leads to consider the dissimilarities between population maps of different tastants not as the result of interanimal variability but instead as the consequence of functionally distinct activated areas that could be used for taste modality discrimination.

The analysis of response overlap across the four taste modal- 

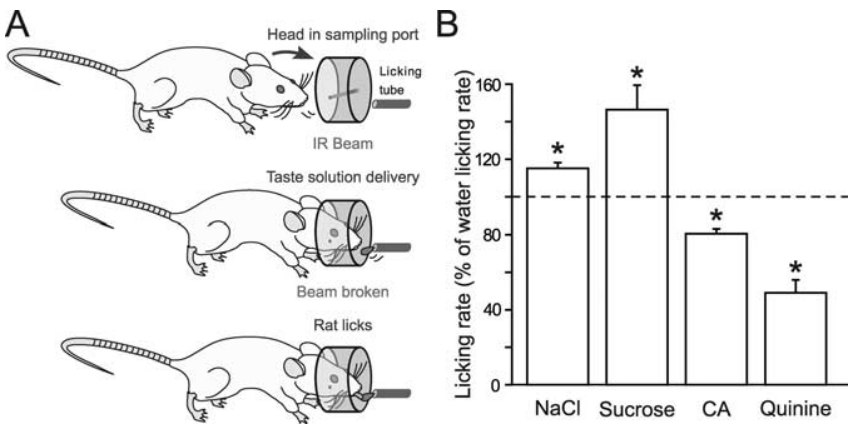

Figure 6. Behavioral assessment of hedonic value associated to different tastants. $\boldsymbol{A}, 0$ rganization of a taste preference trial. The rat's head first enters the sampling port, breaks an infrared (IR) beam and begins to lick the sampling spout. After $1 \mathrm{~s}$, the taste solution is delivered for $500 \mathrm{~ms}$. The frequency of licking is monitored throughout the trial. The next trial is initialized at least $2.5 \mathrm{~s}$ after the end of the delivery of the last stimulus, and the tastants are presented in a random manner with at least one water trial between two different taste stimuli. $\boldsymbol{B}$, Behavioral preference to the four compounds used at the same concentrations as in the imaging experiment. The histograms represent the relative average licking pattern across subjects ( $n=$ 6) obtained from the responses of the single rat across five experimental sessions ( 250 trials for each session). The result is obtained as a ratio between stimulus and water response (dotted line indicates a ratio of 1 ). We averaged across a time window of $1 \mathrm{~s}$, right after the stimulus presentation. Note the significant increase of the licking rate with respect to the baseline $(1 \mathrm{~s}$ period before stimulus application) for $500 \mathrm{~mm}$ sucrose and $500 \mathrm{~mm} \mathrm{NaCl}$ and the decrease for $20 \mathrm{~mm}$ quinine and $10 \mathrm{~mm}$ citric acid (*at least $p<0.005$, all statistics with paired $t$ test analysis). Error bars indicate SEM.

ities revealed the emergence of two groups of stimuli, related to what can be defined as the appetitive (or hedonic) value of the stimulus itself. In fact, higher overlap values were found between $\mathrm{NaCl}$ and sucrose, associated with good nutrients, or between quinine and citric acid, associated with noxious or unripe substances (Fig. 5D). This may suggest a possible additional level of spatial organization representing tastant hedonic value: a pattern for attractive and a pattern for aversive stimuli. Does this activity pattern in the GC really match with taste preference behavior, considering the same stimuli and concentrations used for the imaging experiments? To address this question, we developed a behavioral task to quantify the reactions of rats $(n=6)$ to these taste solutions ( $500 \mathrm{~mm} \mathrm{NaCl}, 500 \mathrm{~mm}$ sucrose, $10 \mathrm{~mm}$ citric acid, $20 \mathrm{~mm}$ quinine, and water). After $1 \mathrm{~s}$ of licking baseline, we delivered a small quantity of a taste stimulus for $500 \mathrm{~ms}$ and measured the change in the licking rate (Fig. 6A). To evaluate the preference for a given taste, the licking patterns were normalized to the water licking pattern. An increase or decrease of the licking rate after taste presentation with respect to the baseline was used to classify a stimulus as attractive or repulsive (in comparison with water). Most importantly, sucrose and $\mathrm{NaCl}$ (at $500 \mathrm{~mm}$ ) show a significant increase in the licking rate with respect to the baseline (average from 0.5 to $1.5 \mathrm{~s}$ poststimulus onset, $\mathrm{NaCl}$, $115 \pm 3 \%, p<0.005$; sucrose, $146 \pm 13 \%, p<0.001$ ), whereas applications of citric acid and quinine cause a significant decrease in the licking rate (citric acid, $80 \pm 2.6 \%, p<0.0008$; quinine, $49 \pm 7 \%, p<0.0007$ ) (Fig. $6 B$ ). This behavioral task confirms that $500 \mathrm{~mm}$ sucrose is attractive and that $10 \mathrm{~mm}$ citric acid and 20 $\mathrm{mm}$ quinine are aversive. It also shows that short presentations of $500 \mathrm{~mm} \mathrm{NaCl}$ are attractive, at least to a water-deprived animal (Scalera, 2000). The dynamics of the behavioral response are faster (Halpern and Tapper, 1971) with respect to the intrinsic signal, and it was not possible to stimulate in the same way in the two experiments (imaging and behavior). However, the temporal evolution of the intrinsic signal is quite stereotyped (Fig. 4C), and, as described by the linear model analysis, the activation maps are not dependent on the time window of integration in the first $4 \mathrm{~s}$ of the response (see supplemental material, available at www.jneurosci.org). Therefore, it was possible to associate the positive or negative hedonic value of the tastant (behaviorally established in the above-noted time window) with the cortical patterns elicited by each tastant in the imaging experiments. Together, the imaging results suggest that the information on tastant hedonic value, which is determined earlier in the gustatory pathway (Grill and Norgren, 1978), is spatially kept at the level of the GC.

\section{Discussion}

Spatial representation of taste modalities accommodates for both segregated and distributed activity

Using in vivo optical imaging, we demonstrated that each taste modality is specifically represented by activation of localized domains in the rat GC. The activated domains for the four basic taste modalities are evident from the examples shown in Figure $5 A$. The patterns are highly reproducible across animals (Fig. 5B). These regions present both segregated and common areas testifying how both segregated and distributed activity contribute to the spatial representation of each tastant.

Interestingly, we also observed a small response to distilled water, located caudally within the GC (Figs. $2 \mathrm{~B}, 4 \mathrm{E}$ ). This is in agreement with recent electrophysiological GC studies, suggesting that water might be considered as an independent taste modality (de Araujo et al., 2003; Stapleton et al., 2006), characterized by specific activation of a cortical area within the GC. However, it is still unclear whether the animals perceive water as another taste modality or are just sensing changes in osmolarity of the salivary solution. Yet, this water-activated GC area might be part of the brain circuitry involved in regulating liquid intake and body homeostatic processes.

\section{Evidences for segregation (or labeled-line)}

In the mammalian gustatory system, activation of different cell types on the tongue has been shown to be sufficient to drive specific taste behaviors (Zhao et al., 2003; Mueller et al., 2005), strongly supporting the existence of a labeled-line model for taste coding. Anatomical and genetic tracing studies also provided evidence of segregated pathways for taste processing from the periphery to the brain in both insects and rodents (Wang et al., 2004; Sugita and Shiba, 2005), confirming that basic organizing principles of sensory processing are conserved among different species. In Drosophila, axon labeling experiments showed that projections from peripheral taste neurons to the brain are segregated by organ and therefore by position of the taste stimulus. Projections from every taste organ are also segregated according to the different taste modality (Wang et al., 2004). An imaging study demonstrated the functional segregation of bitter and sweet qualities at the first relay of the fly brain (Marella et al., 2006). In rodents, a genetic approach (Sugita and Shiba, 2005) suggested a segregation of bitter and sweet and umami projections up to the cortical level. In humans, imaging by functional magnetic resonance the insular cortex, where the primary GC is located (Small et al., 2003), indicated a certain degree of spatial organization. Another study provided the evidence of different patterns of activity in the human primary GC elicited by the five taste modalities, although some overlap existed and the interindividual topographical variability was considerable (Schoenfeld et al., 2004).

The functional spatial patterns that we observed indicate that there is a fundamental difference in the neural representation of bitter and sweet at the cortical level (Fig. 5A, top). Quinine is 
represented more caudally, whereas sucrose activates a more rostral region of the gustatory cortex. Although reflexes of acceptance and avoidance might not need the presence of the GC (Grill and Norgren, 1978), our results provided the evidence for functional taste differentiation even at the cortical level, although the territories are not strictly distinct. Our results are in agreement with previous electrophysiological studies (Yamamoto et al., 1985,1989 ) and partially consistent with the genetic tracing study (Sugita and Shiba, 2005) because the labeled neurons might belong to the regions that we found activated by one but not by the other taste modality. However, the presence of overlapping regions does not allow us to state that the two modalities have a segregated cortical representation. The same considerations hold for any couple of the four stimuli: differential patterns of activation (Fig. $5 A$, bottom; $B$ ), might partly be the result of a labeledline processing from the periphery but does not lead to a completely segregated cortical representation. Our results also contrast with a previous imaging study in guinea pig insular cortex that reported that salt and sweet taste representations are completely segregated (Yoshimura et al., 2004). However, the conclusions of this report must be treated with caution because the authors did not present any quantification of the responses at a population level.

In general, if the one-by-one map comparison seems to reveal regions of specificity of one stimulus to another, the overlay of the four average activation maps shows that there is no apparent region dedicated to the processing of only one taste modality.

\section{Evidence for distributed activity}

Electrophysiological recordings of mammalian GC neurons provide results that are not consistent with segregated pathways of gustatory processing. Recordings from the primary gustatory areas of awake monkeys (Scott and Plata-Salaman, 1999; Verhagen et al., 2004) clearly showed that taste-responsive cells were broadly tuned across modalities (i.e., the same cell responds to several taste modalities and to several properties of the oral stimuli), thus confirming the results found in both anesthetized and awake rodents (Ogawa et al., 1994; Hanamori et al., 1998; Katz et al., 2001; Stapleton et al., 2006). In the macaque GC, Scott and Plata-Salaman (1999) plotted the location of each neuron as a function of its most effective basic stimulus and found no evidence of chemotopic organization of the cortical structure. However, these results do not allow ruling out of the possibility of a spatial organization. Indeed, Yamamoto et al. (1985) proposed a possible chemotopic organization of the rat GC based on extracellular recordings and on an arbitrary grid partition of the cortical area.

We found overlapping regions between the patterns observed in rodent GC, and this is consistent with the above-described electrophysiological data. We hypothesize that, in these commonly activated regions, the incidence of broadly tuned neurons is higher than in the segregated regions. In summary, our functional imaging experiment provided useful data to interpret both electrophysiological and genetic tracing results and to create a bridge between the two apparently contrasting theories of taste coding.

\section{Spatial coding of concentration dependence}

Many studies examined the effect of changes in tastant concentrations on neuronal responses throughout the gustatory pathway. Behavioral studies in both humans and rodents (Bartoshuk, 1974; Spector et al., 1993) found a direct relationship between the perceived intensity and the increasing $\mathrm{NaCl}$ concentration. Elec- trophysiological studies with rodents or nonhuman primates have shown that increasing the stimulus concentration would usually produce an increase in the firing rate. At the GC level, our results are consistent with the majority of both behavior and electrophysiological experiments (Yaxley et al., 1990; Scott et al., 1991) (but see Stapleton et al., 2006). In fact, we measured the responses to three increasing $\mathrm{NaCl}$ concentrations, and we found a monotonic increase in the maximum amplitude and in the size of the response (Fig. $4 B$ ). The expanded size of the activated area (Fig. 4A) might reflect the recruiting of additional neuronal population, probably less specific to the tastant identity and encoding other properties of the gustatory stimulus such as its palatability.

\section{Is there a spatial representation of tastant hedonic value in primary GC?}

The gustatory system is the ultimate control, leading to the decision on which substances will be ingested and which will be rejected. Generally, sweet and salt tastes are associated with nutrients important for survival, whereas bitter and sour tastes are often associated with potentially noxious or unripe substances (Saper et al., 2002). Therefore, a relevant part of gustatory processing is eventually dedicated to the distinction between palatable and unpalatable stimuli.

In rodents, it has been suggested that the process of establishing the palatability (or hedonic value) of a tastant mostly takes place at the level of lower brainstem centers, the nucleus of solitary tract in the medulla and the parabrachial nucleus in the pons (Grill and Norgren, 1978; Kiefer and Orr, 1992). In humans, the behavioral choice among tastes can be made in the absence of the gustatory cortex (Adolphs et al., 2005). However, electrophysiological recordings showed that some GC neurons respond specifically to taste stimulus palatability (Yamamoto et al., 1989). In addition, electrical stimulation of GC increases the palatability of various tastants (Cubero and Puerto, 2000), providing another evidence that GC is involved in the response to stimulus hedonic value.

Our imaging experiments suggest that the primary GC keeps some information related to tastant hedonic value in terms of spatial patterns of activation. In fact, we found that the higher degree of overlap between maps could be related to similar hedonic value of the stimuli (Figs. $5 D, 6 B$ ). This may indicate the existence of some distinct GC territories (enclosing the subregions specific to different modalities) dedicated to the processing of tastant hedonics.

In summary, by in vivo optical imaging of intrinsic signals, we found that the tastants activated spatial areas in the rat GC specific to each modality and that could therefore be essential for tastant discrimination. We also observed that the patterns had more common regions according to the similar tastant hedonic value, proposing a possible way for the GC to keep track of tastant palatability.

\section{References}

Adolphs R, Tranel D, Koenigs M, Damasio AR (2005) Preferring one taste over another without recognizing either. Nat Neurosci 8:860-861.

Bakin JS, Kwon MC, Masino SA, Weinberger NM, Frostig RD (1996) Suprathreshold auditory cortex activation visualized by intrinsic signal optical imaging. Cereb Cortex 6:120-130.

Barnett EM, Evans GD, Sun N, Perlman S, Cassell MD (1995) Anterograde tracing of trigeminal afferent pathways from the murine tooth pulp to cortex using herpes simplex virus type 1. J Neurosci 15:2972-2984.

Bartoshuk LM (1974) NaCl thresholds in man: thresholds for water taste or $\mathrm{NaCl}$ taste. J Comp Physiol Psychol 87:310-325.

Bonhoeffer T, Grinvald A (1991) Iso-orientation domains in cat visual cortex are arranged in pinwheel-like patterns. Nature 353:429-431. 
Bosking WH, Kretz R, Pucak ML, Fitzpatrick D (2000) Functional specificity of callosal connections in tree shrew striate cortex. J Neurosci 20:2346-2359.

Cechetto DF, Saper CB (1987) Evidence for a viscerotopic sensory representation in the cortex and thalamus in the rat. J Comp Neurol 262:27-45.

Chandrashekar J, Hoon MA, Ryba NJ, Zuker CS (2006) The receptors and cells for mammalian taste. Nature 444:288-294.

Contreras RJ, Catalanotto FA (1980) Sodium deprivation in rats: salt thresholds are related to salivary sodium concentrations. Behav Neural Biol 29:303-314.

Cubero I, Puerto A (2000) Electrical stimulation of the insular cortex induces flavor-preferences in rats. Brain Res 872:134-140.

de Araujo IE, Kringelbach ML, Rolls ET, McGlone F (2003) Human cortical responses to water in the mouth, and the effects of thirst. J Neurophysiol 90:1865-1876.

Frank ME (1991) Taste-responsive neurons of the glossopharyngeal nerve of the rat. J Neurophysiol 65:1452-1463.

Frostig RD, Lieke EE, Ts'o DY, Grinvald A (1990) Cortical functional architecture and local coupling between neuronal activity and the microcirculation revealed by in vivo high-resolution optical imaging of intrinsic signals. Proc Natl Acad Sci USA 87:6082-6086.

Grill HJ, Norgren R (1978) Chronically decerebrate rats demonstrate satiation but not bait shyness. Science 201:267-269.

Grinvald A, Lieke E, Frostig RD, Gilbert CD, Wiesel TN (1986) Functional architecture of cortex revealed by optical imaging of intrinsic signals. Nature 324:361-364.

Grinvald A, Shoham D, Shmuel A, Glaser DE, Vanzetta I, Shtoyerman E, Slovin H, Wijnbergen C, Hildesheim R, Sterkin A, Arieli A (1999) Invivo optical imaging cortical architecture and dynamics. In: Modern techniques in neuroscience research (Windhorst $\mathrm{U}$, Johansson $\mathrm{H}$, eds), pp 893-969. Berlin: Springer.

Halpern BP, Tapper DN (1971) Taste stimuli: quality coding time. Science 171:1256-1258.

Hanamori T, Kunitake T, Kato K, Kannan H (1998) Responses of neurons in the insular cortex to gustatory, visceral, and nociceptive stimuli in rats. J Neurophysiol 79:2535-2545.

Katz DB, Simon SA, Nicolelis MA (2001) Dynamic and multimodal responses of gustatory cortical neurons in awake rats. J Neurosci 21:4478-4489.

Katz DB, Simon SA, Nicolelis MA (2002) Taste-specific neuronal ensembles in the gustatory cortex of awake rats. J Neurosci 22:1850-1857.

Kiefer SW, Orr MR (1992) Taste avoidance, but not aversion, learning in rats lacking gustatory cortex. Behav Neurosci 106:140-146.

King CT, Travers SP, Rowland NE, Garcea M, Spector AC (1999) Glossopharyngeal nerve transection eliminates quinine-stimulated fos-like immunoreactivity in the nucleus of the solitary tract: implications for a functional topography of gustatory nerve input in rats. J Neurosci 19:3107-3121.

King CT, Deyrup LD, Dodson SE, Galvin KE, Garcea M, Spector AC (2003) Effects of gustatory nerve transection and regeneration on quininestimulated Fos-like immunoreactivity in the parabrachial nucleus of the rat. J Comp Neurol 465:296-308.

Kosar E, Grill HJ, Norgren R (1986) Gustatory cortex in the rat. I. Physiological properties and cytoarchitecture. Brain Res 379:329-341.

Marella S, Fischler W, Kong P, Asgarian S, Rueckert E, Scott K (2006) Imaging taste responses in the fly brain reveals a functional map of taste category and behavior. Neuron 49:285-295.

Masino SA, Kwon MC, Dory Y, Frostig RD (1993) Characterization of functional organization within rat barrel cortex using intrinsic signal optical imaging through a thinned skull. Proc Natl Acad Sci USA 90:9998-10002.

Mueller KL, Hoon MA, Erlenbach I, Chandrashekar J, Zuker CS, Ryba NJ (2005) The receptors and coding logic for bitter taste. Nature 434:225-229.

Ogawa H, Ito S, Murayama N, Hasegawa K (1990) Taste area in granular and dysgranular insular cortices in the rat identified by stimulation of the entire oral cavity. Neurosci Res 9:196-201.

Ogawa H, Hasegawa K, Ohgushi M, Murayama N (1994) Changes in prop- erties of neuronal responses in two cortical taste areas in rats of various ages. Neurosci Res 19:407-417.

Remple MS, Henry EC, Catania KC (2003) Organization of somatosensory cortex in the laboratory rat (Rattus norvegicus): evidence for two lateral areas joined at the representation of the teeth. J Comp Neurol 467:105-118.

Rolls ET (2005) Taste, olfactory, and food texture processing in the brain, and the control of food intake. Physiol Behav 85:45-56.

Rubin BD, Katz LC (1999) Optical imaging of odorant representations in the mammalian olfactory bulb. Neuron 23:499-511.

Saper CB, Chou TC, Elmquist JK (2002) The need to feed: homeostatic and hedonic control of eating. Neuron 36:199-211.

Scalera G (2000) Taste preference and acceptance in thirsty and rehydrated [correction of dehydrated] rats. Physiol Behav 71:457-468.

Schoenfeld MA, Neuer G, Tempelmann C, Schussler K, Noesselt T, Hopf JM, Heinze HJ (2004) Functional magnetic resonance tomography correlates of taste perception in the human primary taste cortex. Neuroscience 127:347-353.

Scott K (2005) Taste recognition: food for thought. Neuron 48:455-464.

Scott TR, Plata-Salaman CR (1999) Taste in the monkey cortex. Physiol Behav 67:489-511.

Scott TR, Plata-Salaman CR, Smith VL, Giza BK (1991) Gustatory neural coding in the monkey cortex: stimulus intensity. J Neurophysiol 65:76-86.

Simon SA, de Araujo IE, Gutierrez R, Nicolelis MA (2006) The neural mechanisms of gustation: a distributed processing code. Nat Rev Neurosci 7:890-901.

Small DM, Gregory MD, Mak YE, Gitelman D, Mesulam MM, Parrish T (2003) Dissociation of neural representation of intensity and affective valuation in human gustation. Neuron 39:701-711.

Smith DV, St John SJ (1999) Neural coding of gustatory information. Curr Opin Neurobiol 9:427-435.

Spector AC, Grill HJ, Norgren R (1993) Concentration-dependent licking of sucrose and sodium chloride in rats with parabrachial gustatory lesions. Physiol Behav 53:277-283.

Stapleton JR, Lavine ML, Wolpert RL, Nicolelis MA, Simon SA (2006) Rapid taste responses in the gustatory cortex during licking. J Neurosci 26:4126-4138.

Sugita M, Shiba Y (2005) Genetic tracing shows segregation of taste neuronal circuitries for bitter and sweet. Science 309:781-785.

Verhagen JV, Kadohisa M, Rolls ET (2004) Primate insular/opercular taste cortex: neuronal representations of the viscosity, fat texture, grittiness, temperature, and taste of foods. J Neurophysiol 92:1685-1699.

Wang Z, Singhvi A, Kong P, Scott K (2004) Taste representations in the Drosophila brain. Cell 117:981-991.

Yamamoto T, Matsuo R, Kawamura Y (1980) Localization of cortical gustatory area in rats and its role in taste discrimination. J Neurophysiol 44:440-455.

Yamamoto T, Yuyama N, Kato T, Kawamura Y (1985) Gustatory responses of cortical neurons in rats. II. Information processing of taste quality. J Neurophysiol 53:1356-1369.

Yamamoto T, Matsuo R, Kiyomitsu Y, Kitamura R (1989) Taste responses of cortical neurons in freely ingesting rats. J Neurophysiol 61:1244-1258,

Yamamoto T, Matsuo R, Fujimoto Y, Fukunaga I, Miyasaka A, Imoto T (1991) Electrophysiological and behavioral studies on the taste of umami substances in the rat. Physiol Behav 49:919-925.

Yamamoto T, Shimura T, Sako N, Yasoshima Y, Sakai N (1994) Neural substrates for conditioned taste aversion in the rat. Behav Brain Res 65:123-137.

Yaxley S, Rolls ET, Sienkiewicz ZJ (1990) Gustatory responses of single neurons in the insula of the macaque monkey. J Neurophysiol 63:689-700.

Yoshimura H, Sugai T, Fukuda M, Segami N, Onoda N (2004) Cortical spatial aspects of optical intrinsic signals in response to sucrose and $\mathrm{NaCl}$ stimuli. NeuroReport 15:17-20.

Zhao GQ, Zhang Y, Hoon MA, Chandrashekar J, Erlenbach I, Ryba NJ, Zuker CS (2003) The receptors for mammalian sweet and umami taste. Cell 115:255-266. 